\title{
PICKS: Exploring Post-Disciplinary Knowledge in a University's Urban Sustainability Research Landscape
}

\author{
Charlotte Johnson, MRes, PhD'1 and Martin Zaltz Austwick, MPhys, DPhil²
}

\begin{abstract}
This article presents PICKS (Post dIsCiplinary Knowledge Space), a research mapping prototype developed to support cross-disciplinary research into urban sustainability. PICKS is an interactive map of researchers and their research interests, which combines self-reported and institutionally generated data within a framework that demonstrates the epistemic communities existing across the traditional disciplinary divisions. The force-directed graph visualization, prototyped both in Processing and d3.js, offers a novel way to interrogate cross-disciplinary research interests and identify potential networks for collaboration. This article outlines the development of the prototype by discussing the process of research mapping, and describes the results in the context of strengthening a cross-discipline research environment within a university. The article focuses specifically on the field of urban sustainability research, but the approach offers insights into Mode 2 research and the multidisciplinary programs that are becoming increasingly important as universities tackle the urgent questions of sustainability.
\end{abstract}

\section{Introduction}

Urban sustainability is a research field that targets the juncture between social and natural systems and draws together very different knowledge production and validation methods. By crossing over traditional disciplinary boundaries and bringing together diverse epistemologies, such research has generated a rich literature questioning academia's response to challenges facing contemporary societies. Theorists have argued that knowledge production is evolving, suggesting that an era of confident scientific problem solving has segued into an era of post-normal science, embedded in uncertainty and shaped by ethical concerns over the role of science. ${ }^{1}$ The literature notes an ambivalence as research shifts out of the hierarchical terrain of disciplines, which has been adeptly explored by Gibbons et al. ${ }^{2}$ and Nowotny et al. ${ }^{3}$ through their thesis of
Mode 1 and Mode 2 science. Mode 1 refers to research generated within disciplinary boundaries, validated through internally established epistemologies that give preference to the autonomy of scientists and science. Mode 2, by contrast, is issue-driven and assembles researchers using diverse methods and perspectives to generate research validated in part by research-using communities, such as policy makers or industry. There is tension over whether or not Mode 1, with its internally legitimated research, can generate answers to societies' contemporary concerns and make them accessible to research-using communities. However, the issue-driven research of Mode 2 can be interpreted as demonstrating a worrying loss of academic independence. ${ }^{3}$

Sustainability knowledge has been analyzed as a specific subset of this new breed of post-disciplinary knowledge.
Advocates of this research approach argue that "epistemological pluralism" is needed to "fill gaps in knowledge" exposed by sustainability issues. ${ }^{4}$ These researchers explore the power dynamics between different epistemologies and how institutional structures can reinforce epistemic divides. Studies have examined how this new knowledge can be produced within specific research projects, ${ }^{5}$ within academic institutions, ${ }^{6}$ or at the level of national education systems, ${ }^{7}$ arguing that fundamental reorganization of research institutions is required.

This article adds to this body of work with a study of urban sustainability research within a U.K. university, but it moves beyond a critique of existing institutional structures to focus on the fluid way that researchers classify themselves and their collaborations. Academics may work simultaneously

'Institute for Sustainable Resources, ${ }^{2}$ Bartlett Centre for Advanced Spatial Analysis, University College London, London, United Kingdom. 
on projects that speak to a specific discipline as well as applied projects for specific audiences while contributing to inter-, multi-, and cross-disciplinary projects. In this article, we explore this dynamic by creating a disciplineagnostic research space populated by researchers whose work and research interests are classified not only by institutional categories, but also by topic and methodological approach. We argue that this is a research terrain that operates within established disciplinary parameters, but at the same time can transcend these by letting researchers locate themselves in a deinstitutionalized research field.

Research mapping typically connotes policy makers or research managers prioritizing areas for research investment, rather than a process that supports cross-disciplinarity and epistemological pluralism. By contrast, this research mapping exercise was commissioned by University College London (UCL) specifically to support the cross-disciplinary research agenda that the institution promotes. As discussed in an article in this journal, ${ }^{8}$ UCL ran a three-year project analyzing the benefits of cross-discipline research for urban sustainability and identifying the need for additional institutional support. The PICKS visualization was designed to meet this need and support faculty and administrators who are working to minimize institutional and epistemic divides in the pursuit of research into urban sustainability. The process of developing and testing the tool offers novel insights on how sustainability knowledge can be fostered within academic institutions that accommodate established disciplinary hierarchies, as well as research managers orchestrating new impact-oriented research centers, and also researchers searching for "truly creative research in the borderlands between disciplines."3

\section{Method}

The mapping exercise and visualization design ran for six months between April and November 2013. The objectives of the research mapping brief were to:
- Capture an up-to-date snapshot of current research interests related to cities and resources.

- Integrate normative and critical research across the physical sciences, social sciences, arts, and humanities.

- Enable researchers and managers to navigate this terrain in search of research gaps and opportunities for collaboration.

The process involved diagnostic interviews with six research managers, semi-structured interviews with 19 faculty members who are working on urban sustainability to identify emergent themes, a literature review to create an inclusive and discipline-agnostic framework, a staff survey to populate the research map, and the construction of an interactive tool to visualize the results.

\section{Delineating the Field}

Interviews with academics leading the university's cross-discipline Grand Challenge of Sustainable Cities were conducted and additional urban sustainability researchers identified through networking within the university. The 19 interviews were used to gauge emergent strands of sustainability research across the university and to understand personal strategies used to generate cross-discipline collaborations.

These interviews identified Urban Political Ecology (UPE) and Environmental Justice (EJ) as useful theoretical frameworks capable of drawing together different disciplinary perspectives on urban sustainability. EJ specifically critiques the production of data from the natural and physical sciences, embedding such processes in social and political realities. ${ }^{9}$ UPE adds a focus on technological change and urban transition. We used this scholarship to construct an analytical framework that bridged institutional and disciplinary divisions. We adopted UPE's approach of using typologies to characterize differences between perspectives contained within sustainability ${ }^{10,11}$ and EJ's analysis of knowledge systems shaping urban environmental research production and dissemination. ${ }^{12}$ We created the following labels to delineate research interests and approaches in place of established discipline-specific classifiers:

1. Managing metabolic processes: Normative or positivist research on urban social, technical, or environmental processes or resource cycles

2. Critique of knowledge production: Theory-driven research that critiques how knowledge is deployed to shape urban processes

3. Understanding the past: Research that analyzes history or uses historical trends to understand the present

4. Imagining the future: Research that is exploratory or uses future scenarios

5. Empowerment/inequality: Research that analyzes social inequalities or seeks to empower marginalized groups

These categories draw attention to shared theoretical perspectives and communities of practice, and are used to represent a deinstitutionalized research landscape via PICKS.

\section{Combining Datasets}

Following the construction of a framework, the next step was to understand how existing sources of information held by the university are used by researchers and administrators. We were particularly interested in how different datasets reinforced disciplinary hierarchies or enabled cross-fertilization of research.

UCL has a centralized research information service that uses the Department of Human Resources (HR) data on employment status, financial data on grants, and third-party data on publications; the service also hosts a set of 40 research themes that academics can identify with their particular profiles. Researcher profiles are automatically generated by $\mathrm{HR}$, and researchers can add specific research interests, experience, and publication records. However, only 30 percent of staff spend the 
time to update their research profile in the centralized system. The financial systems' data provides good coverage of current projects, but only researchers named on the grants are listed, rather than all the researchers involved. The interviewees for this project (see Delineating the Field) felt this overrepresented established academics and limited new researchers' ability to raise their profiles or develop new networks. Publication data also provides an option for visualizing research links, but this type of data is prone to underrepresent interdisciplinary and cross-disciplinary research. ${ }^{13}$

Interviews with six research administrators identified the patchy nature of self-reported data as a key problem in getting an overview of contemporary research interests and the expertise needed to orchestrate new cross-discipline research groups around issues such as urban sustainability. Interviews with academics also mentioned the utility of identifying potential crossdiscipline collaborators through online research records and emerging research interests. Comments on the range of engagement with the centralized system suggest that maintaining an online profile relates in part to disciplinary affiliation. Some suggested that the lowresponse levels to the centralized system was due to its clunky nature, while others suggested it was rejected in order to show a more discipline-specific profile on a departmental website; some interpreted the lack of an online presence as evidence of a disengaged autonomy, implying Mode 1 characteristics.

\section{Populating the Research Map}

To create a current and inclusive dataset of researcher interests, we mined the centralized database for information that connected researchers to an interest in sustainable urbanism, resources, or cities. This was supplemented and updated through information about current projects provided on departmental websites, as well as through the staff interviews. These sources were used to generate a list of 295 academics with their research interests manually coded according to the resource or urban issue they studied, as well as the methods, theoretical frameworks, populations, and territories focused on, where this information was available and applicable.

To extend this dataset a survey was sent internally to encourage people to put themselves on the map. The survey asked any academics working on sustainable cities or resource issues to provide up to 12 keywords to describe their current research as well as additional information about research approaches and outputs. The survey returned 184 results, adding 146 to the existing dataset.

The survey was also used to evaluate whether or not this Mode 2 type of deinstitutionalized research map reflected knowledge production at UCL. Respondents were asked if they consider themselves to have a specific discipline and $55(29.7 \%)$ checked the No box. This suggests that UCL has a sizeable minority of researchers working on cities and resources who reject disciplinary affiliation. The survey also asked if respondents used and/or critiqued "sustainability" in their research. Again a sizeable minority responded negatively, with 34.8 percent of respondents rejecting "sustainability" in their work; nonetheless these respondents wanted to be included on a map visualizing research on "sustainable resources for sustainable cities."

The qualitative data helps to interpret these responses. Researchers explicitly affiliated with an applied field, or who combine different disciplines in their own work, preferred not to be assigned to a discipline and rejected institutional classifiers as inadequately describing individual research identities. By contrast, the sustainability label was rejected for epistemic reasons. For some researchers working within a natural science paradigm, sustainability was interpreted as a socially produced constraint on what is scientifically possible and therefore seen as a subject for the social rather than physical sciences. For some researchers from the social sciences, arts, and humanities, sustainability was interpreted as a normative category and rejected as not productive for critique or theoretical development.
These findings support the argument that academics today have a more fluid and negotiated relationship to disciplinary affiliation than has been assumed by models of disciplines as "tribes"14 with institutionalized cultures. The findings confirm a need for models of research cultures that can "take account of the shifting and changing patterns of associations as new areas come into being, and grow and merge with others." ${ }^{15}$ Research mapping can support this dynamic way of classifying research synergies by making a post-disciplinary space that brings together the full range of perspectives, from natural sciences to the arts, and encompasses interdisciplinary research initiatives. PICKS helps to visualize this knowledge space as an interest-oriented rather than disciplinedependent research landscape. It is a step toward a more fluid and dynamic classification of academic research and a tool that supports Mode 2 science.

\section{Creating PICKS}

PICKS was designed to incorporate different ways of classifying research and to identify noninstitutional clusters. It combines different types of data to represent the urban sustainability research field at UCL. The first dataset is the automatically generated information about a researcher's department. The second set is the self-reported data that describes researchers' interests. The third dataset was created by manually coding researchers' interests according to the PICKS framework, including current research on resources and cities. The PICKS framework includes three sets of nodes:

1. Urban resources defined broadly to encompass abiotic and biotic resources, cultural resources (e.g., literature), natural and built environments

2. Environmental Justice classifiers

3. Geographic regions where research is ongoing

\section{Results and Discussion: Navigating the Research Terrain}

The mapping exercise resulted in a subset of 441 academics whose research 


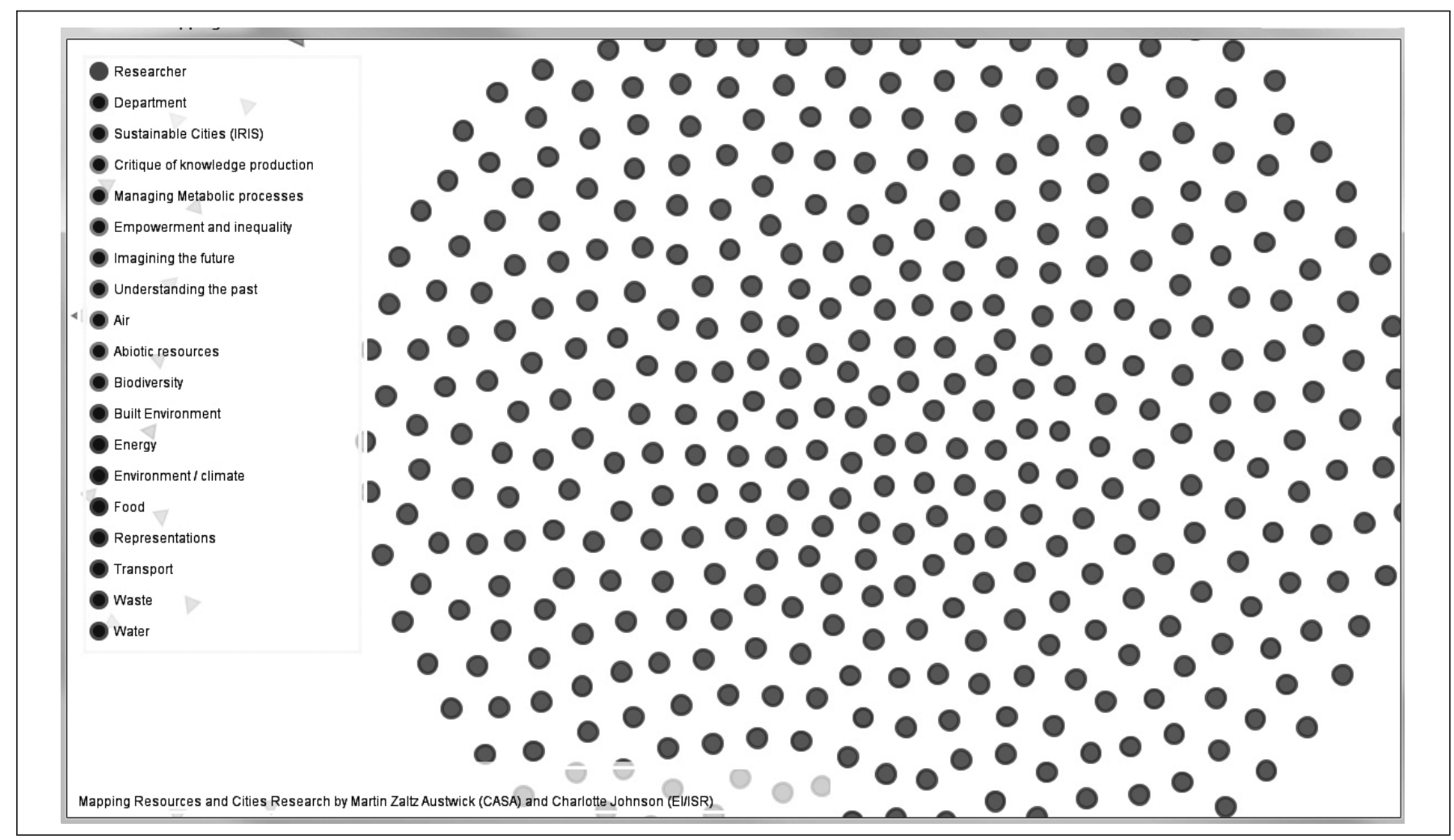

Figure 1. The $441 \mathrm{UCL}$ researchers interested in cities and resources

interests connect them to resources and cities. (See Figure 1.) In PICKS, each researcher is represented as an individual entity and different facets of their research profile are activated as users navigate the terrain and click on nodes to draw together communities of common interest. (See Figures 2 and 3.) The researcher's name is shown by hovering the cursor over the dot; double clicking on the dot opens the researcher's personal profile. In the following images the dark circles represent individual researchers, the triangles are nodes. In PICKS these are different colors to help navigation.

The map is a force-directed graph visualization, ${ }^{16}$ which was built in Processing 2.03 as an app for initial stakeholder discussion. A Processing.js export was used to make the tool webready, but did not have the requisite speed to support the larger number of nodes without significant reductions in framerate. The tool was rewritten using the optimized force layout of D3.js (http://orca.casa.ucl.ac.uk/ martin/ research/PICKS-SUST/PICKS.html) with superior results. Figures 1-4 illus- trate the dynamic clustering produced through this tool, showing research clusters by interest, by department, or both. In Figure 4 the cluster is disaggregated into departments.

PICKS was launched at UCL's symposium, Sustainable Resources for Sustainable Cities in November 2013. Feedback from researchers and research managers in the university, as well as from others outside of the university attending the symposium showed two outcomes.

\section{Outcome 1: Dynamic clustering to identify research synergies and gaps}

PICKS allows researchers to be clustered by department, research interest, or research approach as classified by the UPE-EJ framework (see Delineating the Field). As seen in Figure 5, researchers are clustered by thematic and geographic interest. The nodes Transport, Waste, and Latin America and the Caribbean have been selected, and the map shows the subset of researchers working on any or all of these topics. In this figure, eight people are working on transport in Latin America and the Caribbean, while two people are working on both transport and waste in this region; they can be identified by name in the visualization.

Feedback supported inclusive mapping and suggested that this was useful for identifying researchers outside personal networks. Newer and more junior researchers found PICKS helpful for integrating their own interests into the current field by providing a visual gauge of the relative sizes of clusters, showing underrepresented research themes, and identifying potential collaborators. It also brings more insular academics into the same frame as those actively seeking new collaborators. This was seen as particularly beneficial when designing new products or processes for sustainability because results are "so much stronger if you are talking to people upstream and downstream instead of working in isolation." $[\mathrm{PH}]$

The inclusive aspect was interpreted ambivalently, reflecting the sociology of science literature. Some researchers recognized the democratizing 


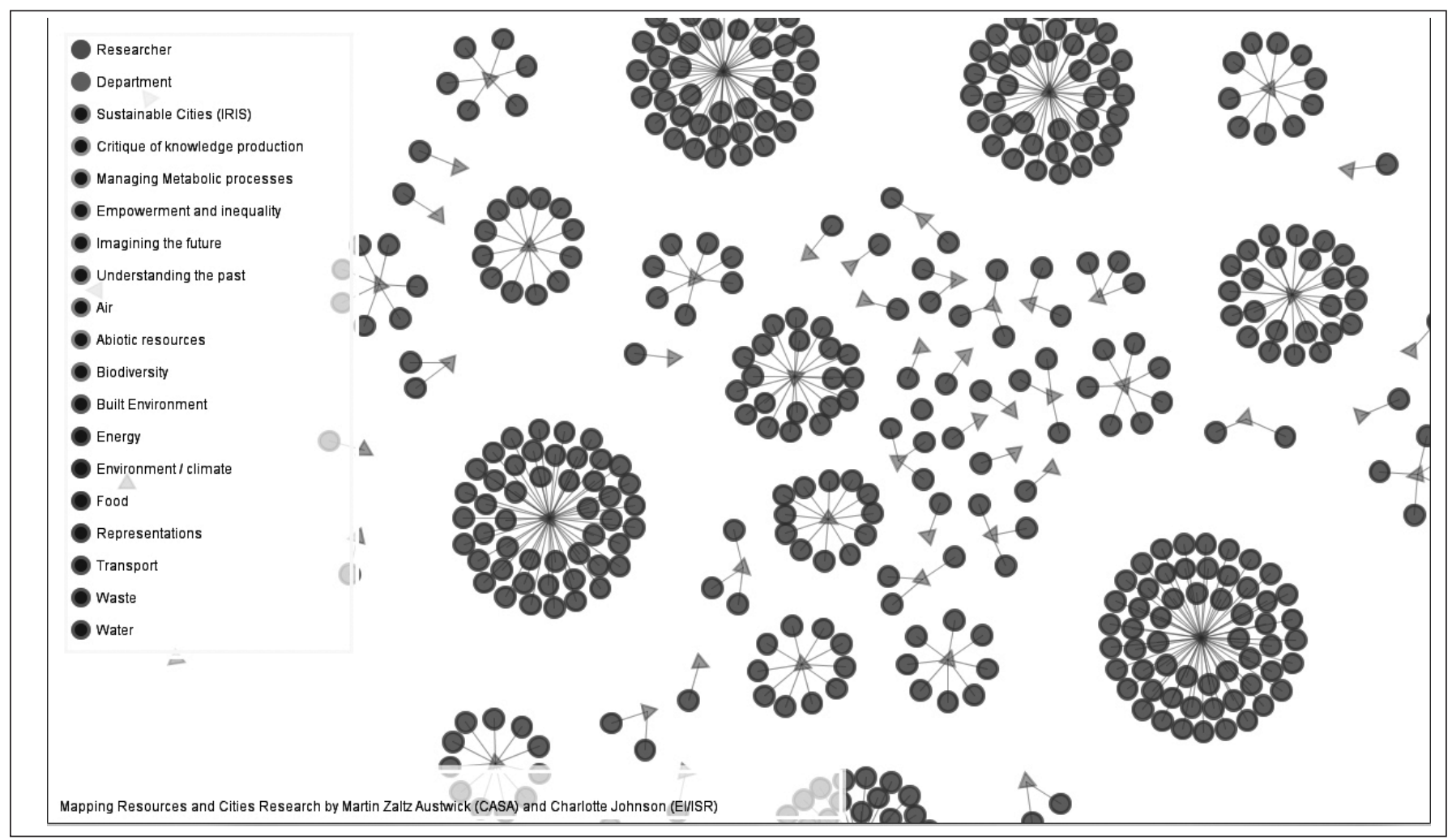

Figure 2. The UCL researchers clustered by department

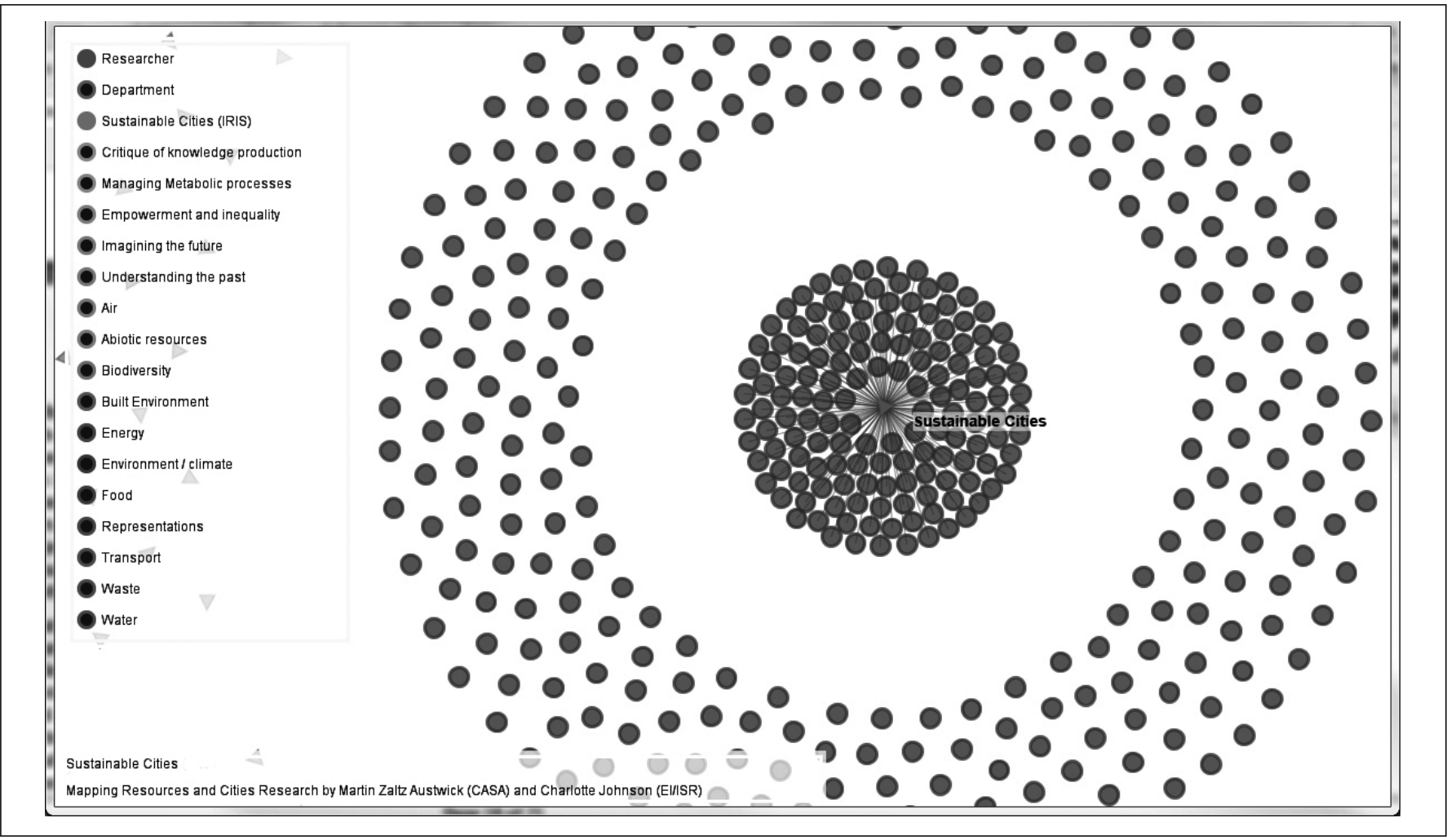

Figure 3. The UCL researchers clustered by interest. The Sustainable Cities node has been activated and researchers who have identified this theme are drawn to the center of the screen. 


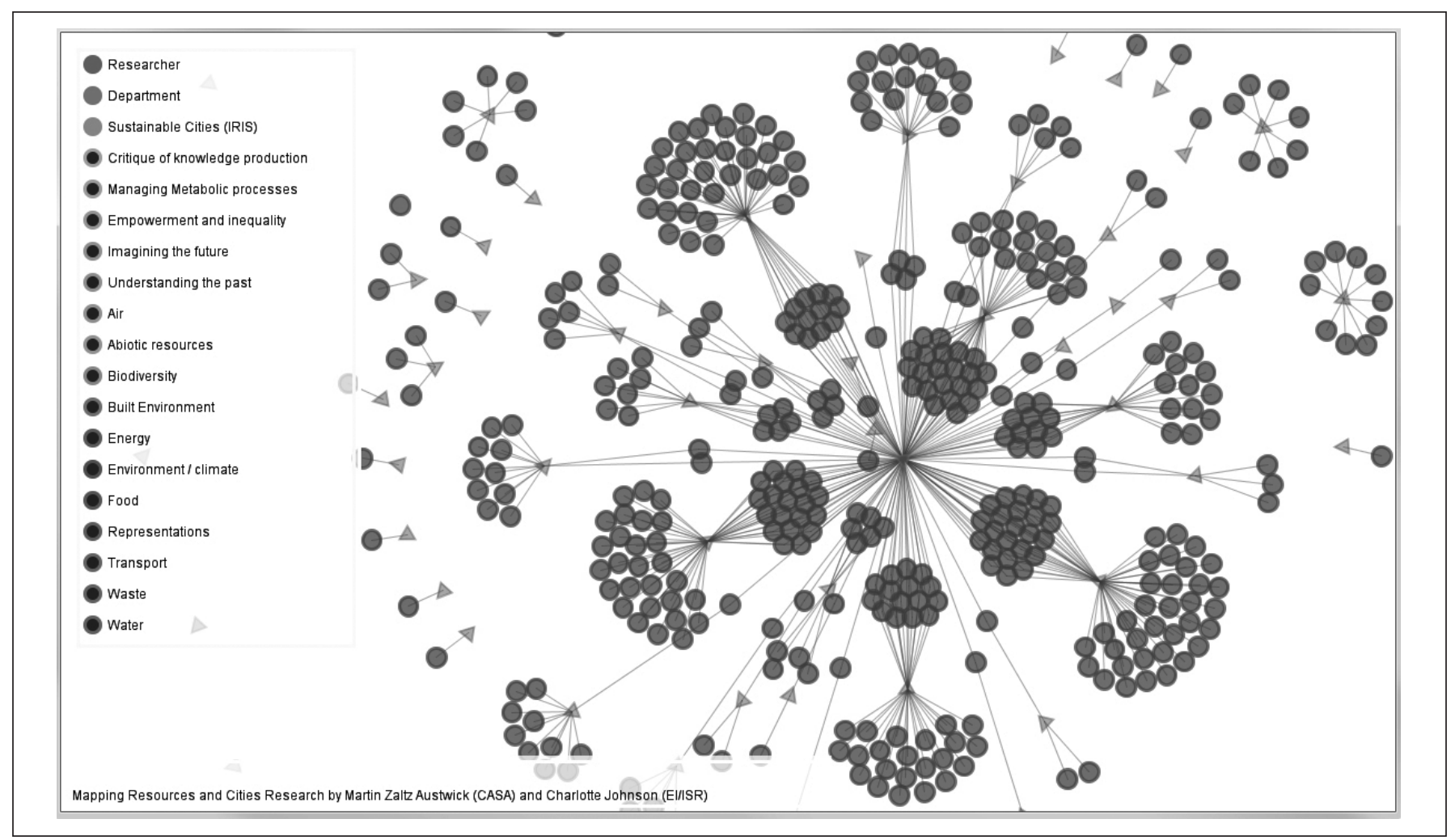

Figure 4. UCL researchers identified by department and by an interest in Sustainable Cities

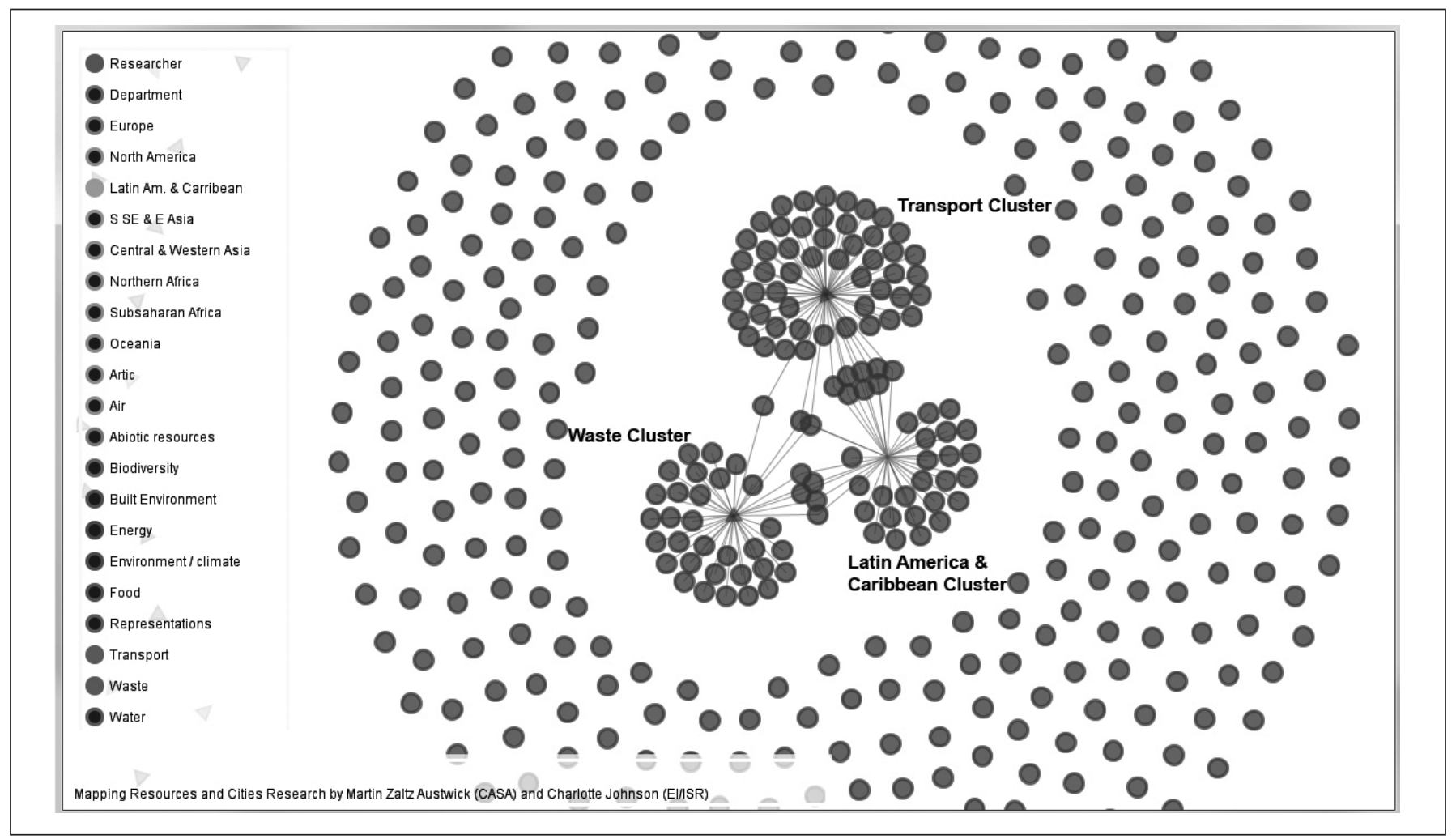

Figure 5. Clustering interest groups 
potential of removing institutionalized hierarchies, while other users felt that experience levels should be incorporated into the map.

Outcome 2: Visually identifying the relative weighting of research themes through the university

PICKS makes it possible to visualize the relative weighting of research themes and approaches across the university. This is useful in identifying epistemic communities outside institutional clustering. In Figure 6 the Sustainable Cities interest node and the Department node are activated, which shows how this topic extends through the different institutions within the university. The greater the proportion of people in a department sharing this interest, the closer the department is to it. In Figure 6 the Development Planning Unit is pulled more closely to the central node, while the Civil, Environmental, and Geomatic Engineering (CEGE) Department remains farther away, despite having a similar number of people working on this topic. This is because CEGE houses a larger proportion of researchers working on cities and resources topics who have not selected Sustainable Cities as a research theme.

Figure 6 also shows that the Sustainable Cities theme is not dominated by any one group; instead a wide spread of departments house researchers connected to this theme. This reflects its status as a centrally supported crossdisciplinary research theme, with associated funding streams. It provides a visual representation of the sentiment, recorded qualitatively, that the university encourages cross-discipline collaborations on sustainability. "Inside UCL, I find it really easy ... there are enough events and structures to facilitate people talking to one another." [ET]

A different landscape is shown in Figure 7. Here departments are clustered around the Energy research theme, and the node is dominated by UCL's Energy Institute, a multidiscipline department established five years ago.
Figures 6 and 7 show visualizations of the different approaches currently used to support cross-discipline researcheither using centralized support to draw researchers from established disciplines (Figure 6) or founding new discipline-agnostic departments that are issue-based (Figure 7).

The EJ classifiers make it possible to further interrogate this cross-discipline landscape by focusing on research approaches, rather than issues. Figures 8 and 9 show how the research landscape is altered by clustering the researchers first around the normative category managing metabolic processes (Figure 8 ) and then around the critique theme (Figure 9). The normative concept of sustainability draws more researchers into the center of the image, while the landscape of Critique is less evenly spread through departments.

Research managers and academics building cross-discipline centers in particular have found this aspect useful. It helps to analyze the spread and rela-

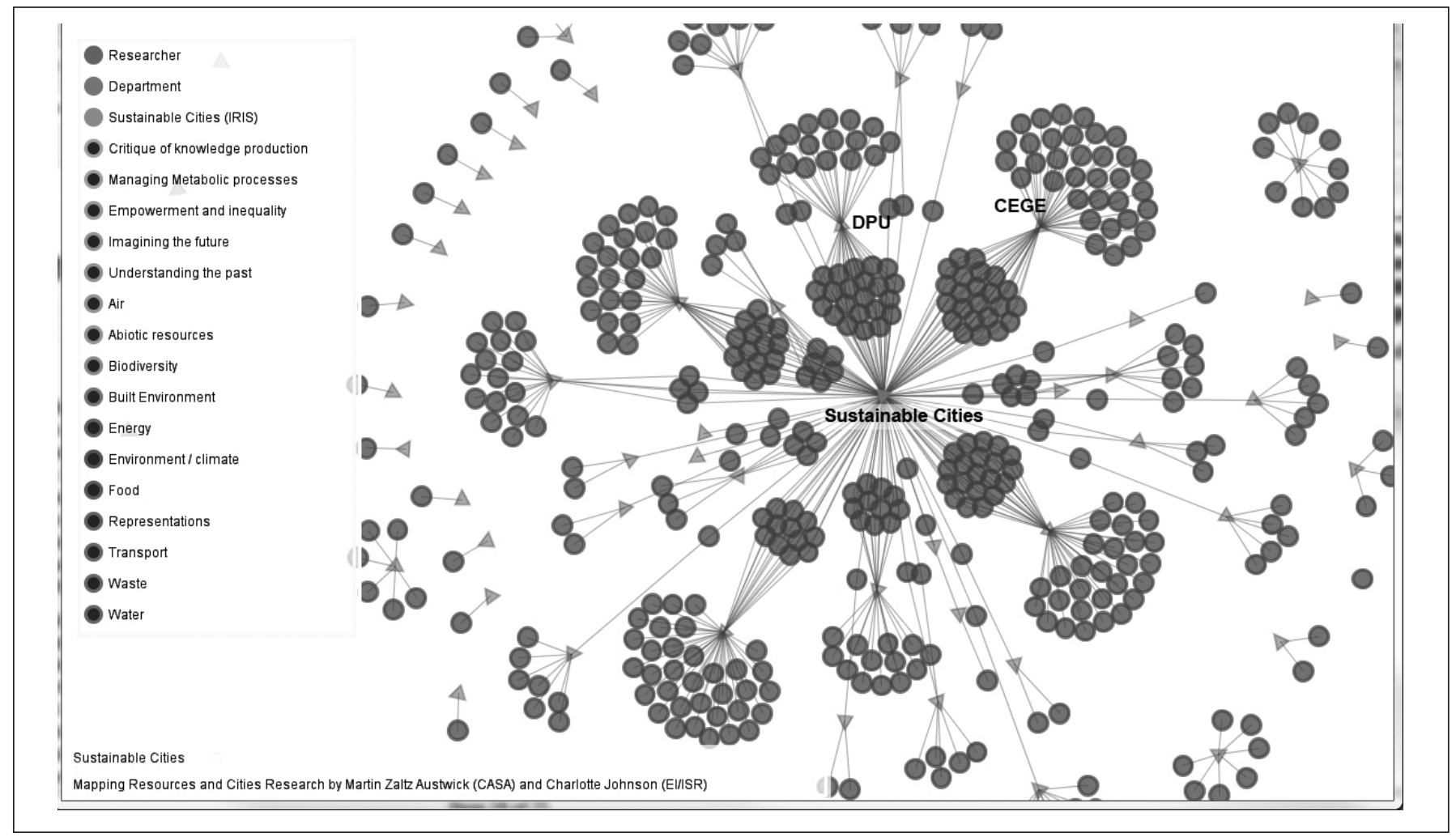

Figure 6. Departmental spread around Sustainable Cities research theme 


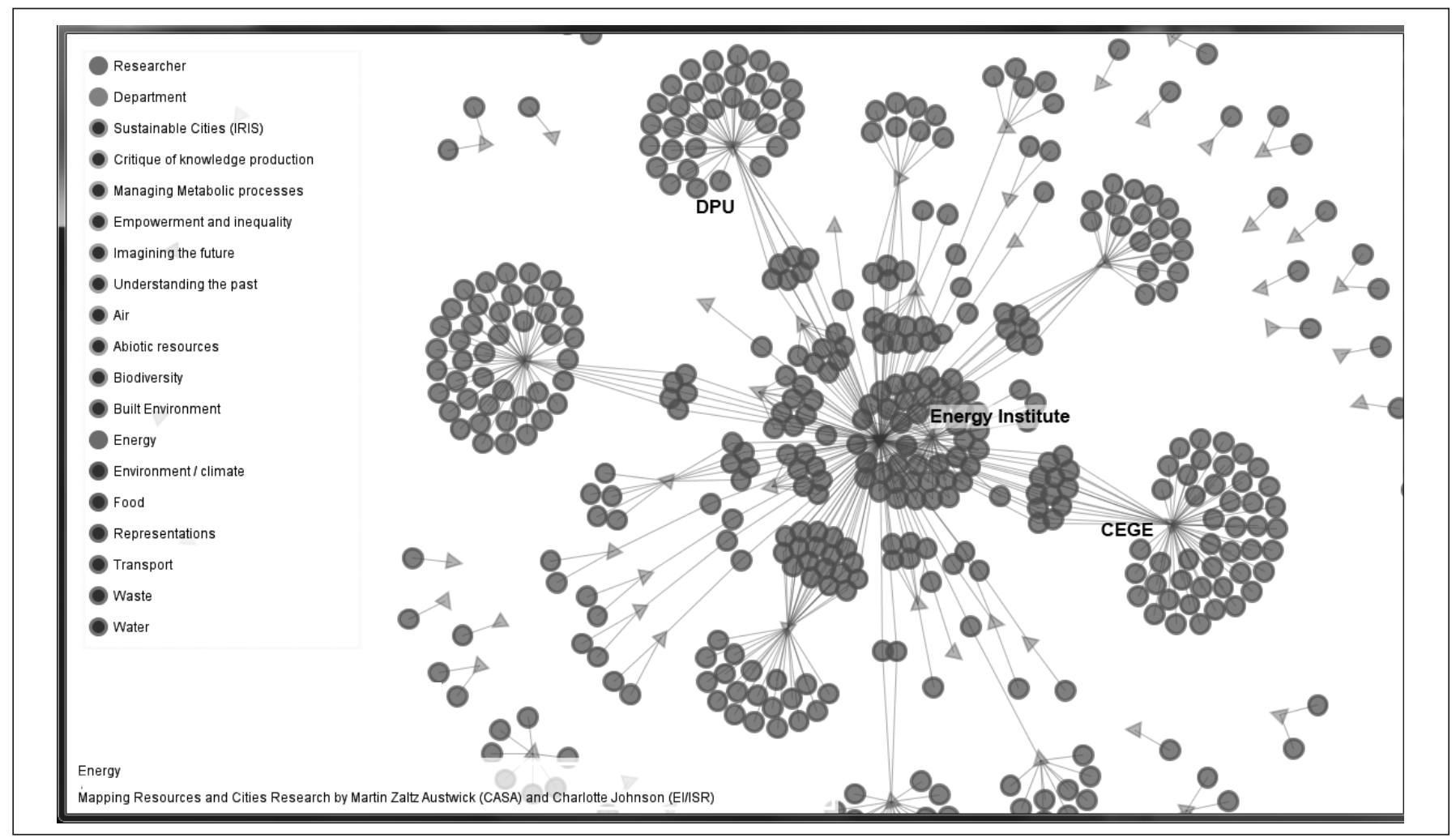

Figure 7. Departmental spread around the Energy research theme

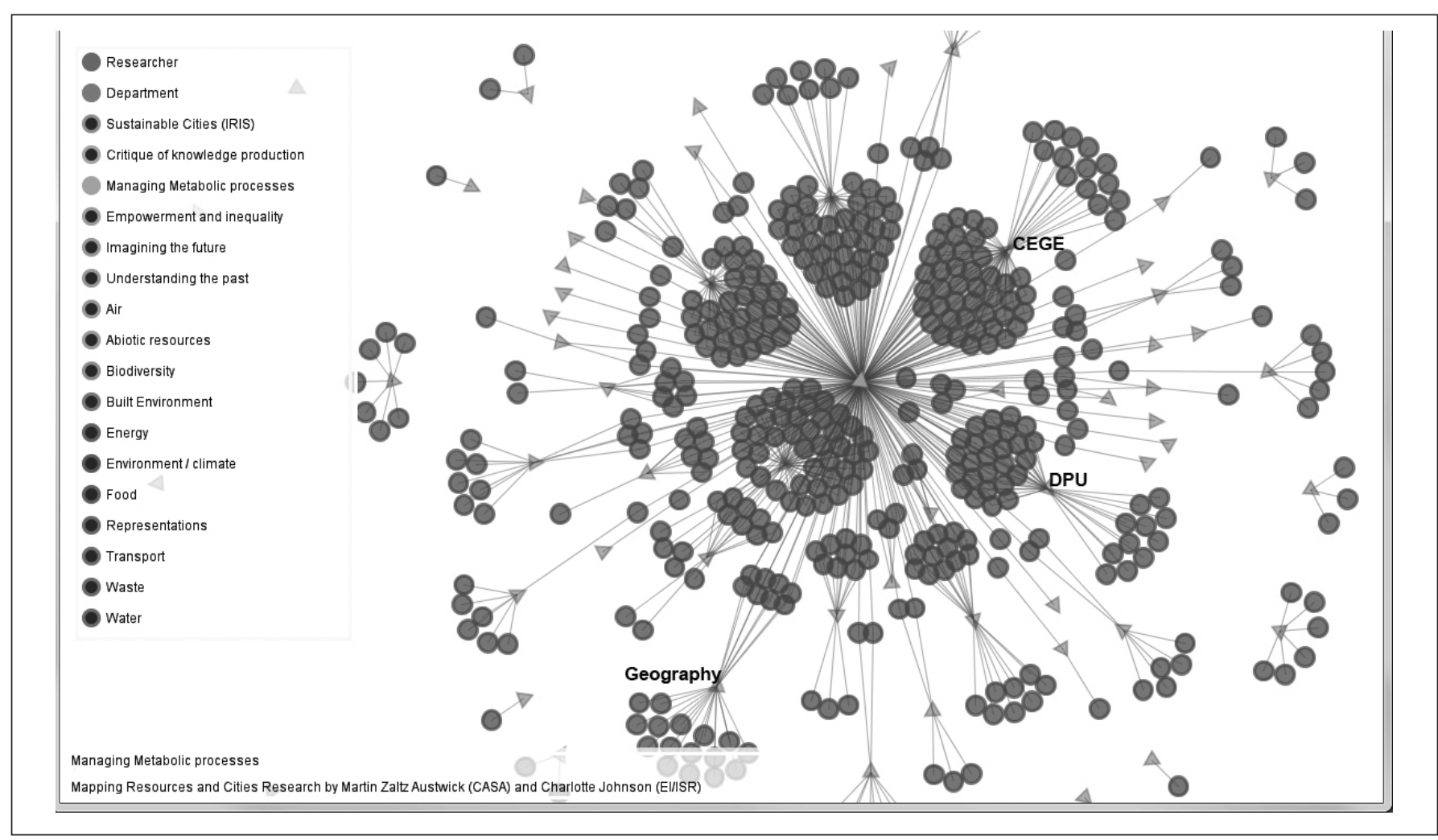

Figure 8. Departmental spread around a normative research paradigm 


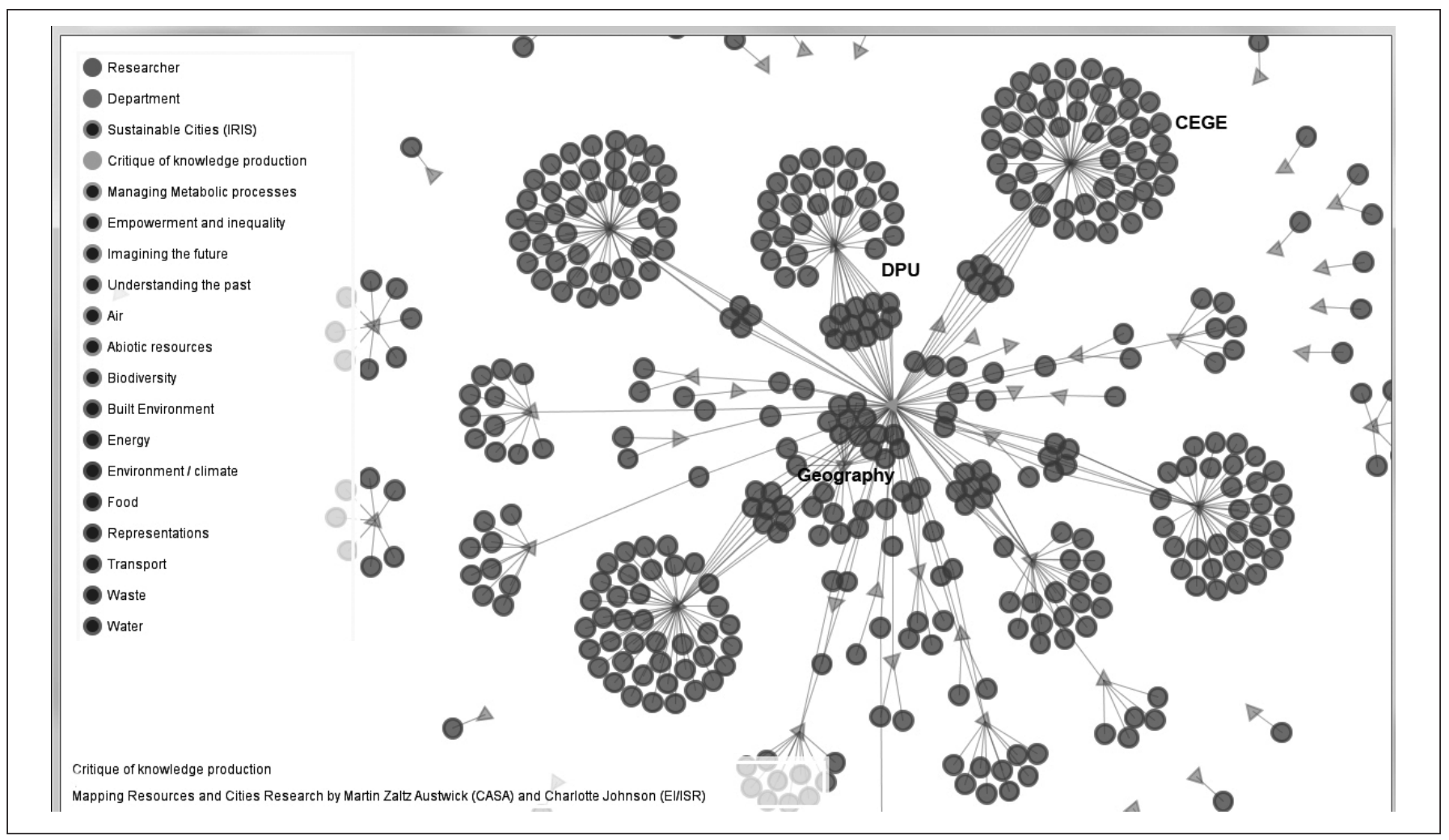

Figure 9. Departmental spread around a critical theory research paradigm

tive weighting of research approaches and perspectives within their centers, or identify academics in other departments working on similar topics. Users commented on the benefit of being able to draw together epistemic communities based on theoretical orientation or methodology, but some found the EJ classifiers confusing and asked for labeling that would be more comprehensible to people working outside an EJ or UPE framework.

\section{Concluding Comments}

Research mapping and visualization can be a step toward reducing entrenched epistemic and organizational constraints in new Mode 2 fields of research such as urban sustainability. We used this methodology to structure a map according to current trends in urban sustainability research, and the approach was successful in getting people from a range of backgrounds to put themselves on the map, even if they rejected institutional or normative classifiers. The utility and the theoretical relevance of PICKS stems from its capacity to enable both internal reflection among the researcher community and external organization by the research management community.

Nowotny et al. ${ }^{3}$ have explained that their thesis of Mode 1 vs. Mode 2 science is a reflection of trends rather than an empirical analysis of knowledge production processes. Our study takes a step toward offering empirical evidence; the survey revealed that a proportion (30\%) of researchers within the university's urban sustainability field do not self-identify with a discipline, but define their work by application. A slightly larger proportion $(35 \%)$ reject the epistemological connotations of being labeled as a "sustainability researcher," but nonetheless interpret their work in relation to this approach. The user response to our interactive mapping tool also supports the dual mode thesis on knowledge production. The deinstitutionalized landscape visualized in PICKS generates an ambivalence, but researchers use it to explore their place in a cross-discipline research landscape and to draw together epistemic or issue-based peer groups. Managers use it to visualize different approaches to support cross-disciplin- ary sustainability research: running centralized activities to draw academics out of discipline-based departments, or developing new centers to dominate a specific research issue such as energy. Where other sociology of science research has focused on restructuring knowledge-producing institutions, our approach has been to capture and visualize some of the Mode 2 research characteristics that operate alongside and across Mode 1 institutional forms. We have shown that visualizing collaboratively designed research maps can support the cross-disciplinary centers and fluid research cultures that are developing through university-based sustainability research, and can support crossdisciplinary approaches to understand real-world problems.

\section{Acknowledgments}

The authors thank James Paskins from UCL's Grand Challenge of Sustainable Cities and Katherine Welch from UCL's Institute for Sustainable Resources for supporting this research, and the interviewees and survey respondents for their contribution. 


\section{Author Disclosure Statement}

No conflicting financial interests exist.

\section{References}

1. Funtowicz SO, and Ravetz JE. The emergence of post-normal science. Sci Polit Moral 1993;17:85-123.

2. Gibbons M, Limoges C, Nowotny H, et al. The New Production of Knowledge: The Dynamics of Science and Research in Contemporary Societies. Sage Publications, Thousand Oaks, CA, 1994.

3. Nowotny H, Scott P, and Gibbons M. Introduction - "Mode 2" revisited: The new production of knowledge. Minerva 2003:41:179-194.

4. Miller TR, and Baird T. Epistemological pluralism: Reorganizing interdisciplinary research. Ecol and Soc 2008; 13 . 5. Longhurst $\mathrm{N}$, and Chilvers J. Interdisciplinarity in Transition? A Technical Report on the Interdisciplinarity of the Transition Pathways to a Low Carbon Economy Consortium. 2012. http://www.lowcarbonpathways.org. uk/lowcarbon/publications/Longhurst_and_Chilvers_2012.pdf (last accessed 5/16/2014).

6. Miller TR, Muñoz-Erickson T, and Redman CL. Transforming knowledge for sustainability: Towards adaptive academic institutions. Int J Sustain in Higher Educ 2011;12:177-192.
7. Schneidewind $U$, and Augenstein K. Analyzing a transition to a sustainability-oriented science system in Germany. Environ Innov Soc Transitions 2012;3:16-28.

8. Paskins J, Bell S, Croxford B, et al. Crossing disciplines to address urban sustainability. Sustain J Record 2012; 5:34-37.

9. Walker G. Environmental Justice: Concepts, Evidence and Politics. Routledge, London, 2012.

10. Davidson K. A typology to categorize the ideologies of actors in the sustainable development debate. Sustain Dev 2014;22:1-14.

11. Arman M, and Davidson K. A typology to position population within sustainability discourse. Local Environ 2014;19:433-448.

12. Agyeman J. Global environmental justice or Le droit au monde? Geoforum 2013. doi:10.1016/j.geoforum.2012.12.021

13. Rafols I, Leydesdorff L, O'Hare A, et al. How journal rankings can suppress interdisciplinary research: A comparison between Innovation Studies and Business \& Management. Res Policy 2012;41:1262-1282.

14. Becher T, and Trowler PR. Academic Tribes and Territories: Intellectual Enquiry and the Culture of Disciplines. Society for Research into Higher Education \& Open University Press, Buck- ingham, England, 2001.

15. Brew A. Disciplinary and interdisciplinary affiliations of experienced researchers. High Educ 2007;56:423-438. 16. Fruchterman TMJ, and Reingold EM. Graph drawing by force-directed placement software. Pract and Exp 1991;21:1129-1164.

Websites for the software:

PICKS-SUST online: http://orca.casa. ucl.ac.uk/ martin/research/PICKSSUST/PICKS.html

Project website: http://www.ucl.ac.uk/ sustainable-cities/results/research-map Source code on GitHub: https://github. com/martinaustwick/PICKS-SUST

Processing software: http://

processing.org

d3 software: http://d3js.org/

Address correspondence to: Charlotte Johnson, MRes, PhD Institute for Sustainable Resources University College London

Central House

Upper Woburn Place London WC1H ONN United Kingdom

E-mail: c.johnson@ucl.ac.uk 